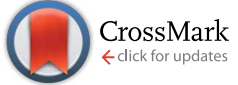

Cite this: RSC Adv., 2014, 4, 32651
Received 26th May 2014 Accepted 16th July 2014

DOI: $10.1039 / \mathrm{c} 4 \mathrm{ra0} 4966 \mathrm{~g}$

www.rsc.org/advances

\title{
Study of Schottky contact in binary and ternary hybrid CdSe quantum dot solar cells
}

\begin{abstract}
M. Ramar, ${ }^{a}$ C. K. Suman, ${ }^{\star a}$ R. Manimozhi, ${ }^{b}$ R. Ahamad ${ }^{a}$ and R. Srivastava ${ }^{a}$
Hybrid binary and ternary organic solar cell devices were fabricated from P3HT-PCBM with CdSe quantum dot materials. The binary and ternary structured solar cells were designed with the combinations of P3HTPCBM (device A), P3HT-CdSe (device B) and P3HT-CdSe-PCBM (device C). The absorption spectra of $\mathrm{P} 3 \mathrm{HT}$, CdSe and PCBM were analyzed in the wavelength range from $350 \mathrm{~nm}$ to $800 \mathrm{~nm}$. The current density-voltage characteristics of the devices were performed in the dark and under illumination to study the conduction process and solar cell performance. The ideality factor of all the devices is more than one. The capacitance-voltage analysis of the devices shows that the depletion width of the binary device $\left(W_{A}\right.$ and $B \sim 39$ and $\left.30.8 \mathrm{~nm}\right)$ is lower than the ternary device $\left(W_{\mathrm{c}} \sim 46.8 \mathrm{~nm}\right)$. The carrier densities of device $A, B$ and $C$ are $1.49 \times 10^{23} \mathrm{~m}^{-3}, 0.52 \times 10^{23} \mathrm{~m}^{-3}$ and $2.6 \times 10^{23} \mathrm{~m}^{-3}$, respectively. The device can be modeled as the combination of two RC parallel circuits (one for the active layer, and the other for the interface) in series with contact resistance $R_{\mathrm{s}} \sim 0$ ohm for device A, B and 60 ohm for device $\mathrm{C}$.
\end{abstract}

\section{Introduction}

Hybrid organic solar cells (HOSCs) based on conjugated polymers, fullerenes, quantum dots, nanowires and quantum tetrapods are new candidates for renewable energy resources because of their advantages of light-weight, flexibility, low-cost, and simple fabrication for large area processing: ${ }^{1-4}$ The blend films composed of poly(3-hexylthiophene) (P3HT) and [6,6]phenyl $\mathrm{C}_{61}$ butyric acid methyl ester (PCBM) have been the center of research since the last decade. The morphology control of the P3HT:PCBM blend has been achieved by thermal annealing, solvent annealing, mixed solvent etc. ${ }^{5-7}$ The morphology control of the binary blend P3HT:PCBM achieved a solar cell power conversion efficiency (PCE) of around $4-5 \%$. The power efficiency established has been well correlated to the good contact of the blend with the electrode. ${ }^{8}$ However, the parameters of binary blend solar cells are difficult to tune further. Blending of multiple donor-acceptor materials with different properties for solar cells is an alternative method. The use of quantum dots for the ternary approach of solar cells is promising due to the possibility of band gap manipulation by size distribution variation of the nano materials. Even near infrared (NIR) band gap materials are viable. ${ }^{9-11}$ Hybrid solar cells of inorganic semiconducting nanoparticles such as $\mathrm{TiO}_{2}$, $\mathrm{ZnO}, \mathrm{CuInS}_{2}$, PbSe, CdSe and CdTe as photo-sensitizers have

${ }^{a}$ CSIR-Network of Institutes for Solar Energy, CSIR - National Physical Laboratory, Dr K. S. Krishnan Marg, New Delhi-110012, India. E-mail: sumanck@mail.nplindia. ernet.in

${ }^{b}$ S S S Academy of Technical Education, Noida, U.P-201301, India been studied, and the use of QDs as additives in the binary solar cells was discussed. ${ }^{12}$ The ternary solar cells with PPV type polymer, PCBM and CdSe nano-particles coated with a trioctylphosphine oxide thin film have shown a maximum PCE of $0.7 \%$ with optimized nano particle size. ${ }^{13}$ Although different combinations of ternary solar cells with quantum dots have been studied, the effect of contact with the electrode in the ternary solar cell approach has not been studied extensively.

In this paper, binary and ternary solar cells were fabricated with P3HT, PCBM and CdSe. The Schottky contacts of these devices have been studied using capacitance-voltage and impedance spectroscopy.

\section{Experimental details}

The hybrid organic solar cells were prepared on commercial indium tin oxide (ITO) coated substrates. The resistivity of the ITO film is $\sim 20 \mathrm{ohm}$ per unit area (from Vin Karola Instruments USA). The ITO coated glass substrates were cleaned in ultrasonic bath with acetone, isopropyl alcohol and D.I. water for 10 min, respectively. The cleaned substrates were dried in an vacuum oven at $120{ }^{\circ} \mathrm{C}$ over 30 minutes. The PEDOT:PSS solution (from Aldrich with 1.3 weight $\%$ in $\mathrm{H}_{2} \mathrm{O}$, conductive grade) was spin coated on ITO substrate and then dried again at $120^{\circ} \mathrm{C}$ for $20 \mathrm{~min}$ in vacuum. The active layer of the solar cells was spin coated on the PEDOT:PSS layer using the blends of P3HT:PCBM ( $1: 0.8$ weight ratio, $30 \mathrm{mg} \mathrm{ml}^{-1}$ ) with $10 \%$ CdSe QD and then dried in vacuum. Many different concentration devices were fabricated but $10 \%$ concentration CdSe device is the optimized device in our lab. All other characterization were done on $10 \%$ 
CdSe device in details. P3HT and PCBM are taken from Aldrich with purity of $99.9 \%$. Oleic acid capped CdSe nanocrystal were synthesized by hot injection method ${ }^{12}$ with nanocrystal size of approximately $4 \mathrm{~nm}$ (calculated by absorption spectra). ${ }^{14}$ Finally; the electrode of $\mathrm{Al}(100 \mathrm{~nm})$ was deposited by thermal evaporation with an active area of $9 \mathrm{~mm}^{2}$ at high vacuum of $10^{-6}$ mbar. The device structures were as

Device A: ITO | PEDOT:PSS $\left(\begin{array}{lll}\sim 50 & \mathrm{~nm}\end{array}\right) \mid$ P3HT-PCBM $(\sim 120 \mathrm{~nm}) \mid$ Al.

Device B: ITO|PEDOT:PSS $(\sim 50 \mathrm{~nm}) \mid$ P3HT-CdSe $(\sim 120 \mathrm{~nm}) \mid$ Al.

Device C: ITO|PEDOT:PSS $(\sim 50 \quad \mathrm{~nm}) \mid$ P3HT-CdSe-PCBM $(\sim 120 \mathrm{~nm}) \mid \mathrm{Al}$.

The device was thermally annealed for 30 minutes at $120^{\circ} \mathrm{C}$ vacuum oven. The photocurrent-voltage characteristics were measured by a source meter (Keithley 2420) in the dark and under illumination. The impedance spectroscopy was performed using an impedance analyzer Solatron 1260 for $100 \mathrm{~Hz}$ to $1 \mathrm{MHz}$ frequency. The oscillation amplitude of the AC voltage was maintained at $50 \mathrm{mV}$ for all measurements.

\section{Results and discussions}

The energy level diagram of the hybrid CdSe blended organic solar cell devices and the absorption spectra of P3HT; PCBM and CdSe nanocrystals taken at ambient condition are shown in Fig. 1(A) and (B). The absorption spectra of P3HT; PCBM and CdSe shows strong broad absorption in the range of $420 \mathrm{~nm}$ to $640 \mathrm{~nm}, 450 \mathrm{~nm}$ to $600 \mathrm{~nm}$ and $600 \mathrm{~nm}$ to $650 \mathrm{~nm}$ respectively. The HOMO level of CdSe is located between the HOMOs of P3HT and PCBM. ${ }^{15}$ The same is true for the LUMO levels. As a consequence, photo induced charge transfer is energetically allowed between P3HT and PCBM, CdSe and PCBM, as well as P3HT and CdSe. The efficient photo induced charge transfer generates positive charge on $\mathrm{P} 3 \mathrm{HT}$ and a negative charge on CdSe and PCBM. The negative charge on CdSe is transferred to PCBM finally to the cathode in case of ternary hybrid solar cells. ${ }^{16}$ At the same time the photons are absorbed by the CdSe at its absorption peak of $\sim 620 \mathrm{~nm}$. The absorption by CdSe QD increases the photon efficiency in the ternary device. Fig. 1(C) shows the atomic force microscopy (AFM) images of active layer used for devices A, B and C just before cathode deposition. All the AFM images was taken using tapping mode of the system (NT-MDT Solver Pro) operation. The root mean square (RMS) surface roughness was found to be $1.78 \mathrm{~nm}, 2.51 \mathrm{~nm}$ and 3.37 $\mathrm{nm}$ respectively. The images of all active layers are smooth that indicates the uniform distribution of CdSe in the polymer matrix. However the surface roughness increases for binary and ternary hybrid system may be due to formation of aggregated domain of nanocrystal. ${ }^{15}$

According to standard emission-diffusion theory, the dependence of current density on voltage for metal-semiconductor rectifying contacts $^{17}$ is given by

$$
\begin{aligned}
& J=J_{0}\left[\exp \left(\frac{q v}{n k T}\right)-1\right] \\
& J_{0}=A^{*} T^{2} \exp \left(\frac{-q \varphi_{\mathrm{B}}}{n k T}\right)
\end{aligned}
$$
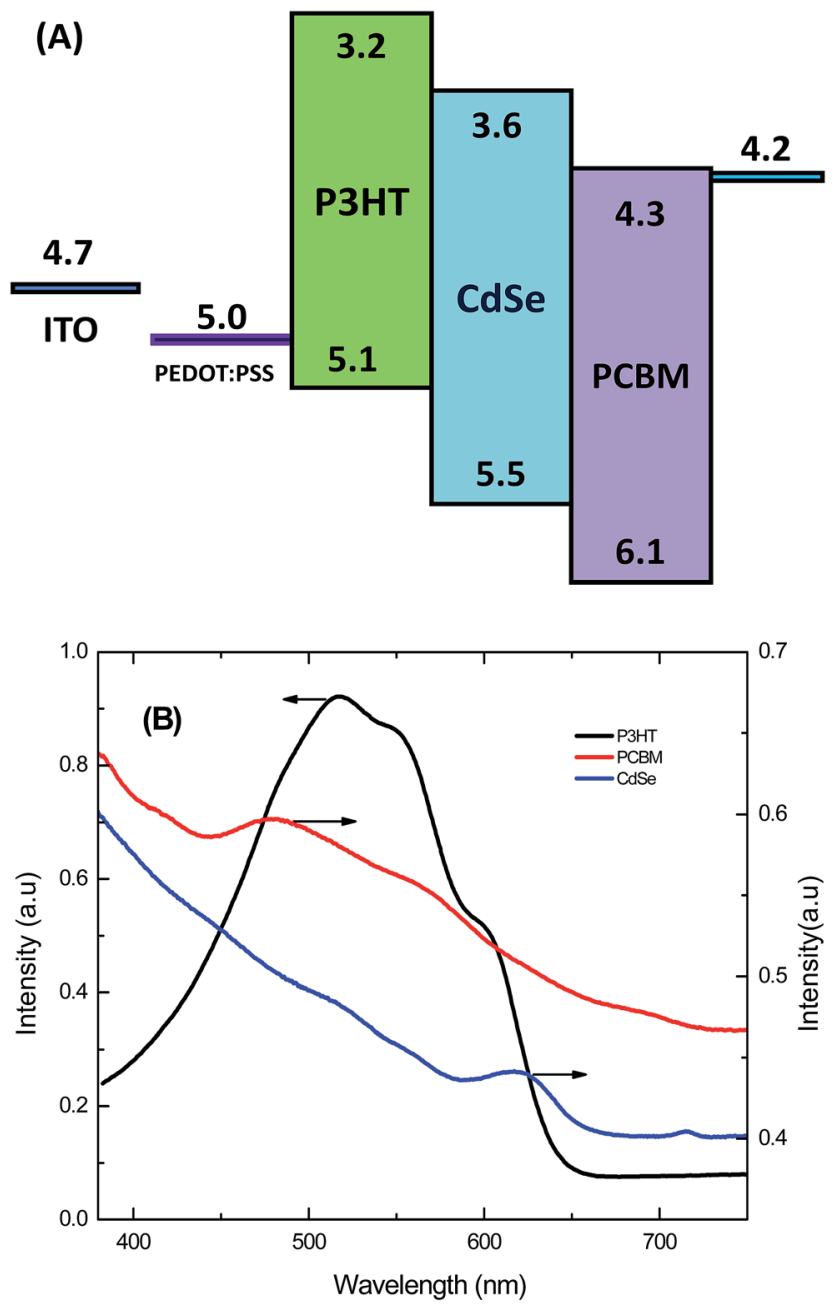

(C)
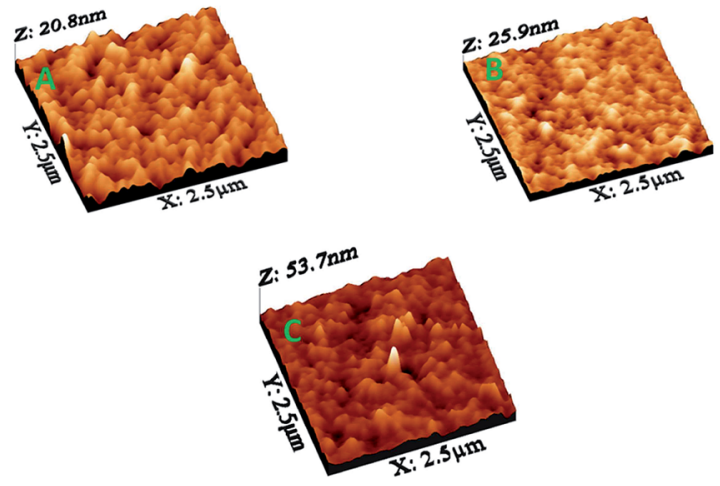

Fig. 1 (A) Energy level diagram of the hybrid CdSe organic ternary solar cells (ref. 15). (B) The absorption spectra of P3HT, PCBM and CdSe measured at ambient condition. (C) The AFM images of active layer used for device A, B and C before cathode layer deposition.

where $A^{*}$ is the effective Richardson constant with $A^{*}=120 \mathrm{~A}$ $\mathrm{cm}^{-2} \mathrm{~K}^{-2}, J_{0}$ is the saturation current density in the absence of external bias, $T$ is the absolute temperature, $n$ is the diode quality factor (ideality factor), $k$ is the Boltzmann constant and $\varphi_{\mathrm{B}}$ is the barrier height. The ideality factors $(n)$ are obtained from the slope. The value of the reverse saturation current density is obtained from the intercepts of $\ln (J)$ versus $V$ at $V=0$. 
Table 1 The device operation parameters (open circuit voltage, short circuit current and fill factor) and the Schottky contacts parameters (built in potential, carrier concentration, depletion width, ideality factor and saturation current density) values of devices A, B and C

\begin{tabular}{lllllllll}
\hline Device & $V_{\mathrm{oc}}(\mathrm{V})$ & $J_{\mathrm{sc}}\left(\mathrm{mA} \mathrm{cm}^{-2}\right)$ & $\eta(\%)$ & $\mathrm{FF}(\%)$ & $V_{\mathrm{bi}}(\mathrm{V})$ & $N_{\mathrm{A}}\left(\times 10^{23} \mathrm{~m}^{-3}\right)$ & $W_{\mathrm{d}}(\mathrm{nm})$ & $n$ \\
\hline A & 0.45 & 5.9 & 0.97 & 37 & 0.79 & 1.49 & 39.07 & 2.7 \\
B & 0.616 & 0.833 & 0.2 & 33 & 0.68 & 0.52 & 0.37 \\
C & 0.49 & 7.1 & 1.2 & 35 & 0.45 & 2.6 & 30.89 & 1.8 \\
& & & & & & &
\end{tabular}

The values of the ideality factor, barrier and reverse saturation current density are listed in Table 1 . The larger than unity value of the ideality factor is attributed to the inhomogeneity of the barrier. ${ }^{18}$ The ideality factor of devices A, B and C are comparable to those of other organic Schottky diodes reported earlier. ${ }^{19}$ The ideality factor reported for donor-acceptor heterojunction of (4,4-bis[ $N$-1-naphthyl- $N$-phenylamino]biphenyl) NPD:(Fullerene) $\mathrm{C}_{60}$ and P3HT:PCBM combinations are 2.6 and 1.8 respectively. The low value of $J_{0}$ for all the device accounted for the larger depletion width (as confirmed from $C-V$ measurements discussed later in this section), which reduces the possibility of tunneling at the interface. The large depletion width causes low charge injection to the electrode and increases the possibilities of recombination of charges again. The effect of large depletion width on the device parameter is decrease of short circuit current and fill factor. However the depletion width has no effect on open circuit voltage. Since all the devices studied here have different light harvesting region so the effect of depletion width in the device parameter is not comparable. It is observed that the forward current also starts increasing sharply at a lower voltage in the device $\mathrm{C}$ in comparison to the device $\mathrm{A}$ and $\mathrm{B}$. However, the reverse saturation current density of the device A is nearly same as the device $\mathrm{C}$. The improvement in the current density of the device $\mathrm{C}$ in comparison to device $\mathrm{B}$ is mainly due to the presence of quantum dot which acts like charge transfer matrix. The CdSe quantum dot helps to change the morphology, because quantum dot provided the platform

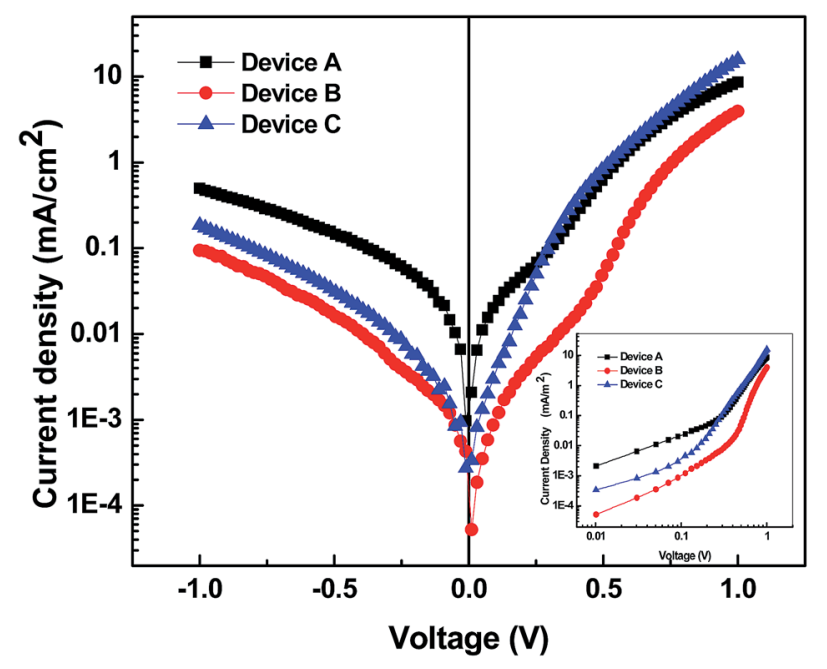

Fig. 2 Semi log of current density versus applied voltage characteristics for device A, B and C in dark. Inset figure shows the log-log plot of current density vs. voltage for all the three devices in dark. for growth of the polymer in a regular nano phase separation manner. ${ }^{12}$ The charge transport mechanism of the Schottky diode can be understood by the logarithmic plot of $J-V$ characteristics as shown in inset of Fig. 2. The curve shows voltage dependence, followed by power law dependence at higher voltages. It is clear from inset of Fig. 2 that the $J-V$ behavior graph has three different linear regions. The first region (voltages up to $0.2 \mathrm{~V}$ ) exhibits an ohmic behavior followed by second region (up to $0.6 \mathrm{~V}$ ) a square law region appears and the charge transport is mainly governed by space charge limited current (SCLC) with an exponential distribution of traps in the band gap of the organic material. ${ }^{20}$ In the third region (at higher voltages), the slope of the curve decreases because of the device approaches the trap filled limit, and behavior in this region is the same as in trap free SCLC. In region 1, the value of current density for device $\mathrm{B}$ and $\mathrm{C}$ decreases that may be because of the CdSe presence. For region 2 and 3, device A and C shows almost same current density, the increased value of current density in device $\mathrm{C}$ is due to the PCBM molecules that makes an energy level ladder for carriers where as for device B the CdSe dominates and have lower value of current in this region too. In case of device B and C; the transport properties may be dominated by the energy traps of CdSe. At the same time the recombination may also be dominated by the presence of traps in CdSe. ${ }^{21}$ The charge transport mechanism of device $\mathrm{B}$ and $\mathrm{C}$ is dominated by traps limited where as for device $\mathrm{A}$ it is space charge limited.

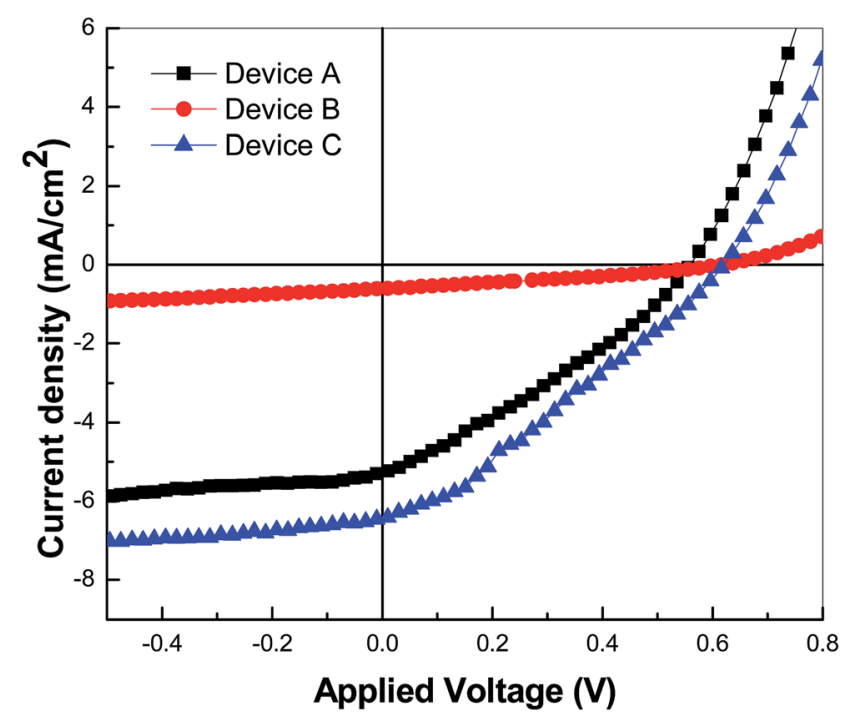

Fig. 3 Current density-voltage characteristics of device $A, B$ and $C$ under illumination at light intensity of $100 \mathrm{~mW} \mathrm{~cm}{ }^{-2}$. 
Fig. 3 shows the current voltage characteristics of the device under air mass 1.5 global (AM1.5G) solar simulator illumination of $100 \mathrm{~mW} \mathrm{~cm}{ }^{-2}$. The open-circuit voltages $\left(V_{\mathrm{oc}}\right)$ are $0.45,0.61$ and 0.49 for device $\mathrm{A}, \mathrm{B}$ and $\mathrm{C}$, respectively. The maximum

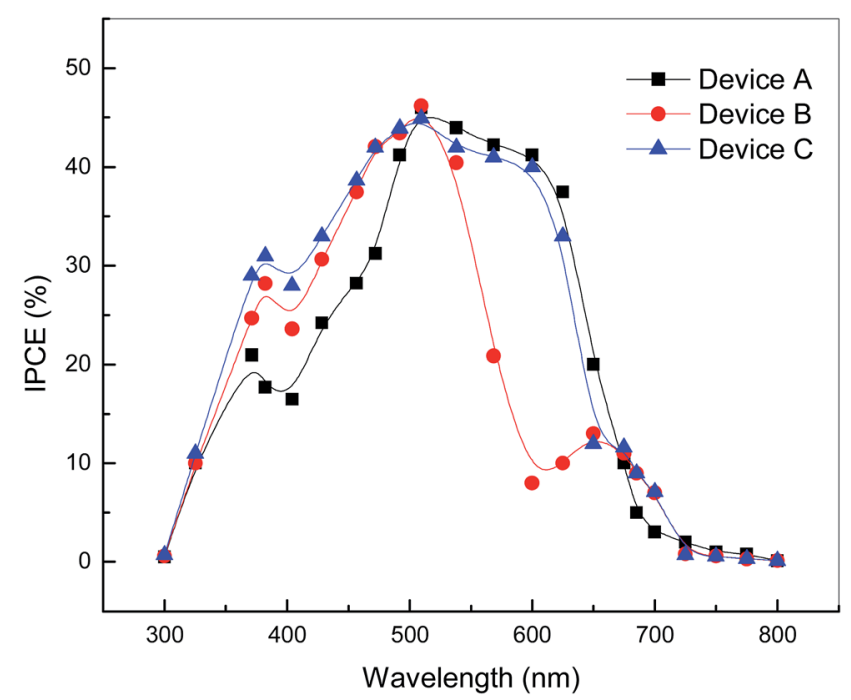

Fig. 4 Incident photon to current conversion efficiency (IPCE) of devices $A, B$ and $C$ measured in the wavelength range 300 to $800 \mathrm{~nm}$. efficiency achieved for device $\mathrm{C}$ is $1.2 \%$ whereas for device $\mathrm{A}$ and $\mathrm{B}$ are $\sim 1 \%$ and $0.2 \%$ respectively. The solar cell efficiency for ternary solar cells (device C) increased by $20 \%$ in comparison to binary device A and more than six times to the device B. The anomalous feature S-shape kink in the current density-voltage characteristics under illumination of the ternary solar cell causes loss of $V_{\mathrm{oc}}, J_{\mathrm{sc}}$ and fill factor too. The S-shape kink may be due to interfacial dipoles, defects and traps. These defects create barriers for carrier extraction..$^{22}$ The solvent additive and mixed solvents may be used in the photoactive layers to overcome these drawbacks by optimizing the microstructure of the ternary blend. The addition of 1,8-diiodooctane (DIO) shows stronger phase separation and enhances P3HT crystallinity leads to fewer distortions in polymer chains in ternary organic solar cells. ${ }^{23}$

Fig. 4 shows the incident photon to current conversion efficiency spectra of device A, B and C. An IPCE peak was observed at $500 \mathrm{~nm}$ and $\sim 650 \mathrm{~nm}$ wavelength which corresponds to the absorption peak of $\mathrm{P} 3 \mathrm{HT}$ and CdSe. The photocurrent response of the active layer for device A, B and C agrees well with the device performance of both binary and ternary system. Fig. 5 shows the capacitance-voltage characteristics of the device A, B and $\mathrm{C}$ measured with AC signal frequency of $100 \mathrm{~Hz}$. The $C-V$ characteristics are related to the interface properties which are related to the interface electronic structures and energy level
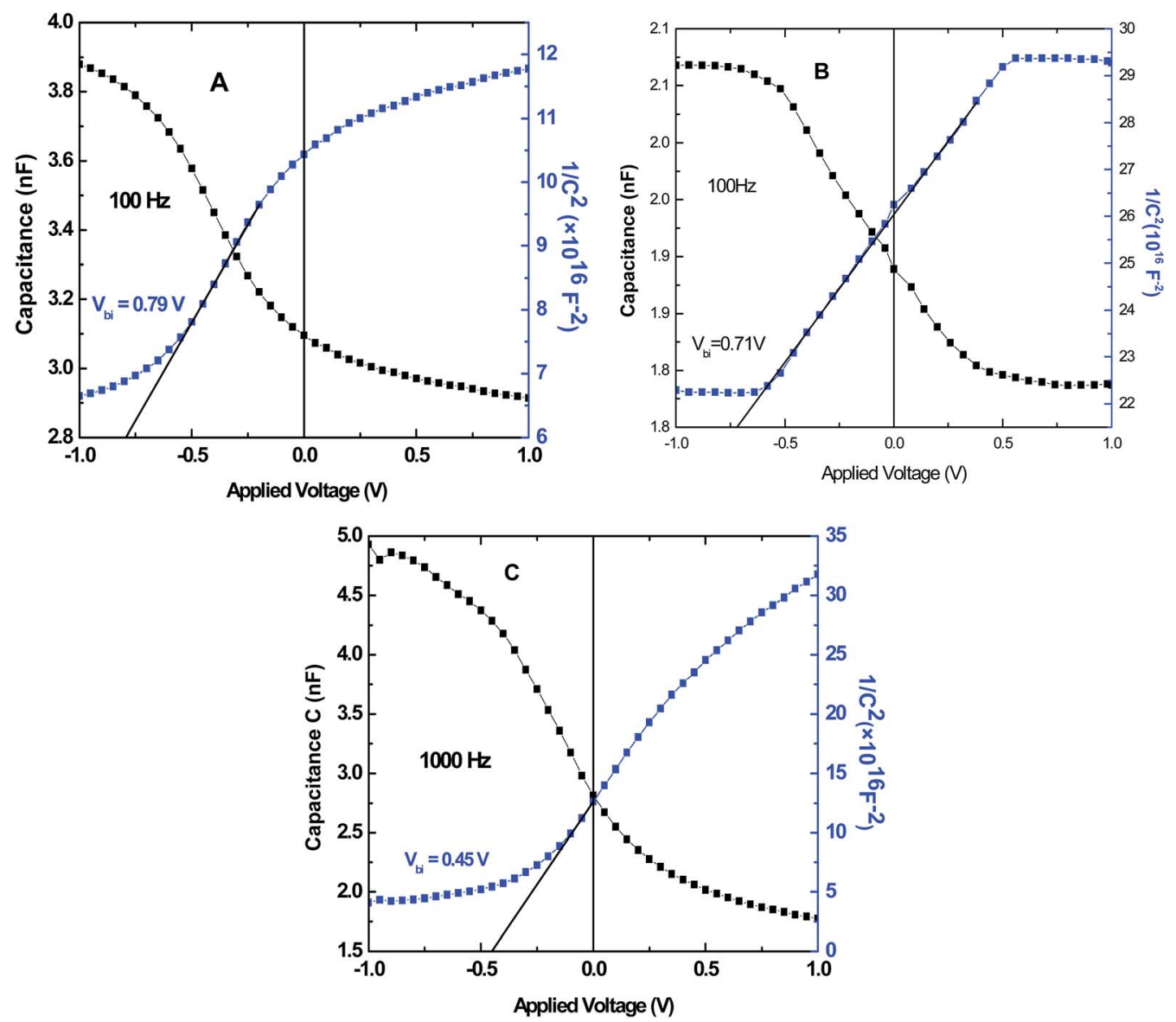

Fig. 5 Variation of Capacitance with applied voltage of device A, B and C and variation of $1 / C^{2}$ with applied voltage. 


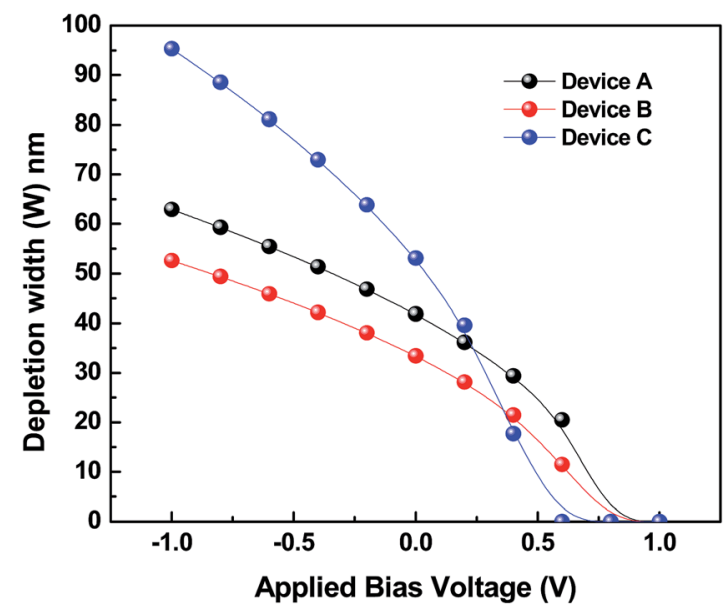

Fig. 6 The variation of depletion width with applied bias voltage of device $\mathrm{A}, \mathrm{B}$ and $\mathrm{C}$. alignment at the junction. The rapid decrease of the capacitance for all devices $\mathrm{A}, \mathrm{B}$ and $\mathrm{C}$ above $-0.5 \mathrm{~V}$ indicates that the injected holes from the ITO begin to be transported from the active material through tunneling, and the tunneling probability increases rapidly with increasing voltage. Fig. 5(b) shows the $1 / C^{2}-V$ characteristics of all the devices A, B and C. The $C-V$ relationship of the Schottky diode ${ }^{24}$ under bias can be expressed as

$$
\frac{1}{C^{2}}=\frac{2 V_{\mathrm{bi}}}{q \varepsilon_{\mathrm{o}} \varepsilon_{\mathrm{s}} N_{\mathrm{A}}}-\frac{2 V}{q \varepsilon_{\mathrm{o}} \varepsilon_{\mathrm{s}} N_{\mathrm{A}}}
$$

where $C$ is the junction capacitance of the Schottky diode per unit area, $V$ is the applied voltage, $\varepsilon_{\mathrm{o}}$ is the free space permittivity, $V_{\mathrm{bi}}$ is the built-in potential at zero bias, $\varepsilon_{\mathrm{s}}$ is the dielectric constant of the material and $N_{\mathrm{A}}$ is the carrier concentration in the depletion layer. The carrier concentration $N_{\mathrm{A}}$ is calculated from the slope of the linear portion of the $1 / C^{2} v s . V$ plot, and the built-in potential $V_{\mathrm{bi}}$ is calculated by extrapolating the linear region of $1 / C^{2}$ vs. $V$ to cut the $V$ axis. The carrier density of devices A, B and C are $1.4 \times 10^{23}, 0.52 \times 10^{23}$ and $2.6 \times$ $10^{23} \mathrm{~cm}^{-3}$ respectively. The carrier concentration of device $\mathrm{A}$
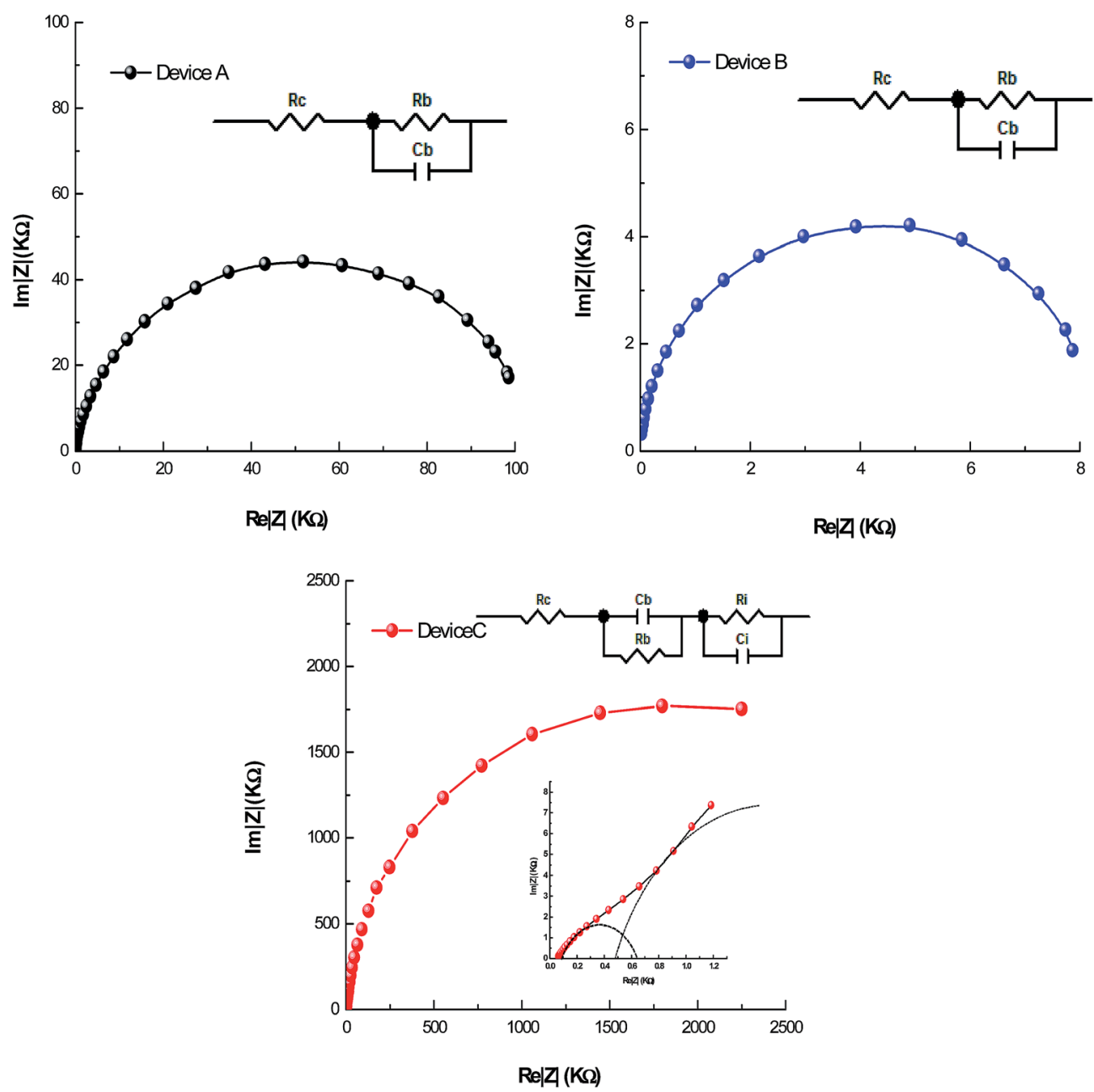

Fig. 7 The cole-cole plot of device $A$ (inset equivalent circuit), $B$ and $C$ (inset equivalent circuit), at applied bias of $0 \mathrm{~V}$, inset of device $C$ figure represents for low value of Real and Imaginary impedance data. 
and $\mathrm{B}$ is lower than device $\mathrm{C}$. The ternary blend device has increased value of carrier concentration than the binary blend device which has good agreement with the $J-V$ plot of all the devices shown in Fig. 2 where the carrier density of device $\mathrm{C}$ is large in comparison to device A and B. The depletion layer width is expressed as $^{\mathbf{1 7}}$

$$
W_{\mathrm{d}}=\left[\frac{2 \varepsilon \varepsilon\left(V_{\mathrm{bi}}-V\right)}{q N_{\mathrm{A}}}\right]^{1 / 2}
$$

The variation in the depletion layer width with the applied voltage, as estimated on the basis of eqn (4), is shown in Fig. 6.

The values of the built-in potential, carrier concentration and depletion layer width are listed in Table 1 . The cole-cole plots of the device at zero bias voltage in dark are shown in Fig. 7. The device A and B shows a single semicircle in the colecole plot with series resistance $\left(R_{\mathrm{s}}\right)$ respectively; this can be modeled as with a combination of resistance and capacitance in parallel. For device $\mathrm{C}$ the cole-cole plot shows double semicircle. Hence the ternary system of the organic device can be modeled as two different system of carrier relaxation. The first region is the active layer and the second region as the interface region. ${ }^{25}$ The equivalent circuit is shown in inset of Fig. 7 representing as the combination of parallel resistance-capacitance $(R-C)$ circuits in series. Each parallel $R-C$ circuit represents the active layer, the interface between the active layer and contact. Since the resistance of the interface $\left(R_{\mathrm{i}}\right)$ are not clearly resolved in the cole-cole plot for the binary solar cells, they are combined as $R_{\mathrm{x}}=R_{\mathrm{b}}+R_{\mathrm{i}}$ in Fig. 7A and $\mathrm{B}$. The impedance and its real and imaginary part of the complete device can be presented as follows

$$
Z=R_{\mathrm{s}}+\frac{R_{\mathrm{b}}+j \omega R_{\mathrm{b}}{ }^{2} C_{\mathrm{b}}}{1+\omega^{2} R_{\mathrm{b}}{ }^{2} C_{\mathrm{b}}{ }^{2}}+\frac{R_{\mathrm{i}}+j \omega R_{\mathrm{i}}{ }^{2} C_{\mathrm{i}}}{1+\omega^{2} R_{\mathrm{i}}{ }^{2} C_{\mathrm{i}}{ }^{2}}
$$

where 'b' and 'i' stand for the bulk or active layer and interface layer. The resistance $\left(R_{\mathrm{x}}\right)$ for binary device $\mathrm{A}$, B is $55 \mathrm{k} \Omega, 4.1 \mathrm{k} \Omega$ respectively and for ternary device $\mathrm{C}$ is $1600.3 \mathrm{k} \Omega$, respectively. The bulk resistance for device $\mathrm{C}$ is $0.3 \mathrm{k} \Omega$. The separate interface resistance for device $\mathrm{C}$ is very high $1600 \mathrm{k} \Omega$. The high resistance may be caused by poor interaction between the polymer and CdSe, with the formation of phase separation and rough and/or heterogeneous morphologies or by a poor charge transfer process and/or poor charge transport. The ternary device is not only having the increased device efficiency but the interface resistance is also present in the device and therefore while designing the ternary organic solar cells the interface resistance has to be considered for improvement in solar cell efficiency. Since the high interface resistance will reduce the carrier flow to the respective electrode and increase the possibilities of recombination of charges. The overall solar cells performances will decrease. The device operation parameters (open circuit voltage, short circuit current and fill factor) and the Schottky contacts parameters (built in potential, carrier concentration, depletion width, ideality factor and saturation current density) values of devices A, B and C are shown in Table 1.

\section{Conclusion}

In conclusion, our work highlights the importance of ternary blend and demonstrated that the device performance of the ternary hybrid solar cells increases in comparison to the same binary solar cells. The interface of the blended binary and ternary active materials to the contact electrode plays very important role for device efficiency. The depletion width is higher in ternary device in comparison to the binary device. The carrier density of devices A, B and C are 1.4, 0.52 and $2.6 \times$ $10^{23} \mathrm{~m}^{-3}$ respectively. The cole-cole plots indicate that the active layer and interface layer of the device can be modeled as the combination of two parallel circuits of resistance and capacitance in series with each other. The contact resistance is very negligible in both types of devices. The active layer resistance is in few kilo ohms in darks for all devices. But the interface resistance of device $\mathrm{C}$ is $1600 \mathrm{k} \Omega$. It has been observed that the ternary blend causes increase in interface resistance that may be due to poor interaction between the polymer and CdSe in ternary blend, rough and heterogeneous morphologies, poor charge transfer process and poor charge transport. The increased interface resistance limits the performance of device by lowering the value of current density and efficiency. Therefore, incorporating a third component is a complex task that needs to address by taking into account many aspect such as energy level, charge transport and charge carrier transfer in these blended system.

\section{References}

1 C. W. Tang, Two-layer organic photovoltaic cell, Appl. Phys. Lett., 1986, 48, 183.

2 W. U. Huynh, J. J. Dittmer and A. Paul Alivisatos, Hybrid Nanorod-Polymer Solar Cells, Science, 2002, 295, 2425-2427.

3 P. V. Kamat, Quantum Dot Solar Cells. Semiconductor Nanocrystals as Light Harvesters, J. Phys. Chem. C, 2008, 112, 18737-18753.

4 Y. Zhou, Y. Li, H. Zhong, J. Hou, Y. Ding, C. Yang and Y. Li, Hybrid nanocrystal/polymer solar cells based on tetrapodshaped $\mathrm{CdSe}_{x} \mathrm{Te}_{1-x}$ nanocrystals, Nanotechnology, 2006, 17, 4041.

5 F. Padinger, R. S. Rittberger and N. S. Sariciftci, Effect of post-production treatment on plastic solar cells, Adv. Funct. Mater., 2003, 13, 85-88.

6 G. Li, V. Shrotriya, J. S. Huang, et al., High efficiency solution processable polymer photovoltaic cells by self-organization of polymer blends, Nat. Mater., 2005, 4, 864-868.

7 J. K. Lee, W. L. Ma, C. J. Brabec, et al. Processing additives for improved efficiency from bulk heterojunction solar cells, $J$. Am. Chem. Soc., 2008, 130, 3619-3623.

8 X. Liu, J. Y. Lee and L. J. Guo, Efficiency and stability enhancement of polymer solar cells using multistacks of C60/LiF as cathode buffer layers, Org. Electron., 2013, 14, 469-474.

9 A. Tang, S. Qu, F. Teng, Y. Hou, Y. Wang and Z. Wang, Recent Developments of Hybrid Nanocrystal/Polymer Bulk 
Heterojunction Solar Cells, J. Nanosci. Nanotechnol., 2011, 11, 9384-9394.

10 J. Tang and E. H. Sargent, Infrared Colloidal Quantum Dots for Photovoltaics: Fundamentals and Recent Progress, $A d v$. Mater., 2011, 23, 12-29.

11 R. Debnath, O. Bakr and E. H. Sergent, Solution-processed colloidal quantum dot photovoltaics: A perspective, Energy Environ. Sci., 2011, 4, 4870-4881.

12 J. N. de Freitas, I. R. Grova, L. C. Akcelrud, E. Arici, N. S. Sariciftcic and A. F. Nogueira, The effects of CdSe incorporation into bulk heterojunction solar cells, J. Mater. Chem., 2010, 20, 4845-4853.

13 H. Fu, M. Choi, W. Luan, Y. S. Kim and S. T. Tu, Hybrid solar cells with an inverted structure: Nanodots incorporated ternary system, Solid-State Electron., 2012, 69, 50-54.

14 W. W. Yu, L. Qu, W. Guo and X. Peng, Experimental Determination of the Extinction Coefficient of CdTe, CdSe, and CdS Nanocrystals, Chem. Mater., 2003, 15(14), 28542860 .

15 R. Ahmad, V. Arora, R. Srivastava, S. Sapra and M. N. Kamlasanan, Enhanced performance of organic photovoltaic device by incorporation of tetrapod-shaped CdSe nanocrystals in polymer-fullerene systems, Phys. Status Solidi A, 2013, 210(4), 785-790.

16 M. Koppe, H.-J. Egelhaaf, G. Dennler, M. C. Scharber, C. J. Brabec, P. Schilinsky and C. N. Hoth, Near IR sensitization of organic bulk heterojunction solar cells: Towards Optimization of the spectral response of organic solar cells, Adv. Funct. Mater., 2010, 20, 338-346.
17 S. M. Sze, Physics of Semiconductor Devices, Wiley Eastern Ltd, New Delhi, 1981.

18 A. K. Singh and R. Prakash, Organic Schottky diode based on conducting polymer-nanoclay composite, $R S C A d v ., 2012,2$, 5277-5283.

19 G. A. H. Wetzelaer, M. Kuik, M. Lenes and P. W. M. Blom, Origin of the dark-current ideality factor in polymer: fullerene bulk heterojunction solar cells, Appl. Phys. Lett., 2011, 99, 153506.

20 J. B. M. Krishna, A. Saha, G. S. Okram, S. Purakayastha and B. Ghosh, Influence of traps on charge transport in metal ion doped polyaniline, J. Phys. D: Appl. Phys., 2009, 42, 115102.

21 J. Albero, E. M. Ferrero, J. Ajuria, C. Waldauf, R. Pacios and E. Palomares, Photo-induced electron recombination dynamics in CdSe/P3HT hybrid hetrojunction, Phys. Chem. Chem. Phys., 2009, 11, 9644-9647.

22 A. Kumar, S. Sista and Y. Yang, Dipole induced anomalous Sshape $I-V$ curve in polymer solar cells, J. Appl. Phys., 2009, 105, 094512.

23 T. Ameri, P. Khoram, J. Min and C. J. Brabec, Organic ternary solar cells: A Review, Adv. Mater., 2013, 25, 4245-4266.

24 A. Watanabe, S. Murakami and K. Mori, Electronic properties of polypyrrole $/ n$-Si heterojunctions and polypyrrole/metal contacts, Macromolecules, 1989, 22, 4231.

25 S. Noh, C. K. Suman, D. Lee, S. Kim and C. H. Lee, Study of buffer layer thickness on bulk heterojunctions solar cell, $J$. Nanosci. Nanotechnol., 2010, 10, 6815-6818. 